Commun. math. Phys. 8, 327-337 (1968)

\title{
On Equal-Time Commutation Relations of Renormalized Currents in Perturbation Theory I
}

\author{
B. SCHROER* \\ University of Pittsburgh, Pittsburgh 13, Pennas. \\ P. STICHeI \\ Deutsches Elektronen-Synchrotron (DESY), Hamburg \\ Received February 15, 1968
}

\begin{abstract}
Equal-time current commutation relations are considered in renormalizable field theories. Renormalized currents are obtained by means of solutions of the Yang-Feldman equations for Heisenberg field operators in perturbation theory. For the computation of matrix elements of current commutators we apply Jost-Lehmann-Dyson type techniques. The equal time limit is taken with the help of symmetrical time-smearing functions which interpolate the $\delta$-function. Our methods avoid any cut-off procedure and lead therefore to unambiguous results. In order to avoid spin complications, our general methods are applied to trilinear resp. quadrilinear couplings of isoscalar and isovector spin 0 -mesons in first order perturbation theory. We find that the zero-space components of the currentcommutator matrix elements behave for small time separation $T$ like $\ln (T)$ $\operatorname{grad}_{x} \delta(\boldsymbol{x}-\boldsymbol{y})$.
\end{abstract}

\section{Introduction}

It was pointed out by the present authors some time ago that the concept of equal-time current commutation relations (ETCR) is compatible with the general principles of quantum field theory [1]. In order to understand the dynamical content of ETCR and their general form allowed within the field theoretic framework it may be helpful to discuss ETCR for renormalizable field theories in perturbation theory. In this paper we take up the discussion of this problem.

The first investigations along this line have been undertaken by JoHnsow and Low [2] and other authors [3]. The procedure used in these papers we want to criticize for two reasons:

1. No explicitly renormalized currents are used.

2. The ETCR $\left[j_{\mu}(x), j_{\nu}(y)\right]_{x_{0}=y_{0}}$ is computed by taking appropriate time limits from the time-ordered product $T\left[j_{\mu}(x), j_{v}(y)\right]$. 3829.

* Supported by the U. S. Atomic Energy Commission under Contract AT(30-1)- 
Now it is well-known that the $T$-product is a non-unique ambiguous quantity even if finite currents are used. In particular, the usual perturbation theoretic definition of the $T$-product of renormalized currents leads always to finite expressions only if the currents under consideration participate in the interaction Lagrangian (i. e. Quantum electrodynamics, Yang-Mills type of theories). In any other theory (the Johnson-Low model, the pseudoscalar meson-nucleon coupling etc.) the so defined $T$-products will diverge in general. Instead of really redefining the $T$-product, JoHnson and Low [2] introduced a causality violating cut-off in momentum space and lift this cut-off after having taken equal times. The interchange of the two limits cut-off $\rightarrow \infty$ and $T \rightarrow 0$ is always questionable, as f. i. by another cut-off procedure (Pauli-Villars regularization) one may kill every non-canonical term in ETCR [3]. In order to avoid such dubious techniques, we first compute renormalized currents by means of iteration solutions of the Yang-Feldman equations for Heisenberg field operators ${ }^{1}$. We derive the Jost-Lehmann-Dyson (JLD) representation for matrix elements:

$$
\left\langle\phi\left|\left[j_{\mu i}\left(\frac{x}{2}\right), j_{v k}\left(-\frac{x}{2}\right)\right]\right| \psi\right\rangle .
$$

where the JLD-spectral functions are represented by parametricintegrals. We then compute ETCR as the limit:

where

$$
\lim _{T \rightarrow 0} \int d x_{0} f_{T}\left(x_{0}\right)\left\langle\phi\left|\left[j_{\mu i}\left(\frac{x}{2}\right), \quad j_{v k}\left(-\frac{x}{2}\right)\right]\right| \psi\right\rangle
$$

$$
\begin{aligned}
f_{T}\left(x_{0}\right) & =\frac{1}{T} f\left(\frac{x_{0}}{T}\right) \text { with } f \in \mathscr{D}, \\
f\left(x_{0}\right) & =f\left(-x_{0}\right) \text { and } \int f_{T}\left(x_{0}\right) d x_{0}=1 .
\end{aligned}
$$

In this paper we restrict ourselves on trilinear and quadrilinear couplings of spin zero mesons. We introduce the following meson fields:

$A_{i}(x)=$ ith component of an isovectorial pseudoscalar meson field.

$B(x)=$ isoscalar scalar meson field.

The following couplings between these fields are renormalizable and lead to a non-trivial isovector-vector current:

$$
\begin{array}{ll}
L_{1}=g_{1} \boldsymbol{A}^{2}(x) B(x) & L_{2}=g_{2} \boldsymbol{A}^{2}(x) B^{2}(x) \\
L_{3}=g_{3} \boldsymbol{A}^{2}(x) \boldsymbol{A}^{2}(x) . &
\end{array}
$$

We may also consider combinations of these couplings like $f$. i.

$$
L_{4}=g_{4}\left(\boldsymbol{A}^{2}(x)+B^{2}(x)\right)^{2} .
$$

1 In this way some results on ETCR in quantum electrodynamics have been obtained recently by LANGERHOLC [4]. 
The isovector-vector current is defined as usual:

$$
j_{\mu i}^{(V)}(x) \equiv Z \frac{1}{4} \varepsilon_{i n m}\left\{A_{n}(x), \stackrel{\leftrightarrow}{\partial_{\mu}} A_{m}(x)\right.
$$

In theories with couplings $L_{1,2,4}$, we may define in addition an isovector-axialvector current, whose most general form (if at most bilinear terms in the fields are allowed) is given as follows:

$$
j_{\mu i}^{(A)}(x)=\frac{a}{2}\left\{B(x), \partial_{\mu} A_{i}(x)\right\}+\frac{b}{2}\left\{A_{i}(x), \partial_{\mu} B(x)\right\}+c \partial_{\mu} A_{i}(x) .
$$

The constants $a$ and $b$ will be fixed in zeroth order by the requirement that ETCR should be as "quark-like" as possible. For certain interactions there is another interesting possibility for fixing these constants: Consider $L_{4}=g_{4}\left(\boldsymbol{A}^{2}(x)+B^{2}(x)\right)^{2}$ then the choice $a=-b$ and $c=0$ i. e. $j_{\mu i}^{(A)}(x) \sim\left\{A_{i}(x), \overleftrightarrow{\partial \mu} B(x)\right\}$ leads in case of equal masses for the $A$ - and $B$-particles to a conserved axial vector current. The reason for this additional law is the fact, that $L_{4}$ bears a higher symmetry than just $S U$ (2). In case of the $L_{3}$ interaction Lagrangian, it has been realized by Kuo and Sugawara [5] and also by one of the present authors (P. STrCHEL, unpublished) that an axialvector-current involving trilinear meson-terms can be constructed. Using the canonical commutation relations formally, one can see that the Ansatz

$$
j_{\mu i}^{(A)}(x)=\alpha \partial_{\mu} A_{i}(x)+\beta\left\{\boldsymbol{A}^{2}(x) \partial_{\mu} A_{i}(x)-2 \boldsymbol{A}(x) \partial_{\mu} \boldsymbol{A}(x) A_{i}(x)\right\}
$$

with $\alpha \beta=-1 / 4$ fulfills the ETCR

$$
\left[j_{0 i}^{(A)}(x), j_{0 k}^{(A)}(y)\right]_{x_{0}=y_{0}}=i \varepsilon_{i k l} \delta(\boldsymbol{x}-\boldsymbol{y}) j_{0 l}^{(V)}(x)
$$

because the trilinear terms commute at equal times with itself and the relative commutator between the linear and the trilinear term yields the wanted vector current. The argument is, however, completely formal since field operators cannot be multiplied at one point. In order to obtain a well defined axialvector current, one must at least take out the short distance singularities of the two point function. For the special case of the free field this leads to the well known Wick product. An explicit computation with the well-defined trilinear Wick product:

$$
: \boldsymbol{A}^{2}(x) \overleftrightarrow{\partial_{\mu}} A_{i}(x) \text { : }
$$

shows the appearance of an additional (divergent) term in the ETCR of the form:

$$
\delta(\boldsymbol{x}-\boldsymbol{y}) \int \varrho(x) d x: A_{i}(x) \overleftrightarrow{\partial_{0}} A_{l_{i}}(x):
$$

where $\varrho=$ two particle phase volume.

Hence, we conclude that a zero order (in the coupling $g_{3}$ ) axialvector current fulfilling ETCR cannot be defined. Within our trilinear Ansatz the only way out of this difficulty is:

and hence $a \sim 1 / g_{3}$.

$$
\beta \sim g_{3}
$$


In that case the axialvector current contains a $g_{3}^{-1}$-order term and the zeroth order ETC would require second order perturbation terms in the meson field. We want to restrict ourselves in this paper only to first order meson fields and hence we will discuss the axialvector problem of $L_{3}$ in a subsequent paper.

We next compute all ETCR between $V$-and $A$-currents in the model characterized by $L_{2}$, restricting ourselves in this paper to first order perturbation theory. Thereby, the ETCR between the zero and space components of the currents which are bilinear in the meson fields turn out to be logarithmic divergent, i. e. $\sim \ln (T) \operatorname{grad} \delta(\boldsymbol{x})$ for $T \rightarrow 0$ independent of the interpolating symmetric test function.

\section{Equal Time Commutators in Zeroth Order and the Form of the Axialvector Current}

It has been noted by several authors [6], that our vector current (1) leads in zeroth order to a $[V, V]$-ETCR, which agrees with Gell-Mann's simple "quark result" [7] only for the isospin antisymmetric combination.

The constants $a, b, c$ in the zeroth order axialvector current:

$$
\begin{aligned}
& j_{\mu i}^{(A)(0)}(x)=a B^{(0)}(x) \partial_{\mu} A_{i}^{(0)}(x)+b A_{i}^{(0)}(x) \partial_{\mu} B^{(0)}(x) \\
& +c \partial_{\mu} A_{i}^{(0)}(x)
\end{aligned}
$$

we will now fix in such a way that for the combination of ETCR antisymmetric in the "internal indices" (i. e. isospin and "kind of current") we obtain Gell-Mann's "quark result" [7].

From the requirement:

$$
\frac{1}{2}\left[j_{0 i}^{(A)}{ }^{(0)}(x), j_{\nu k}^{(A)}{ }^{(0)}(y)\right]_{x_{0}=y_{0}}^{t r}-(i \leftrightarrow k)=i \varepsilon_{i k r} \delta(\boldsymbol{x}-\boldsymbol{y}) j_{\imath r}^{(V)(0)}(x)
$$

we obtain the restriction:

$$
-b^{2}=a \cdot b=1 \text {. }
$$

Then the ETCR $\left[j_{0 i}^{(A)(0)}(x), j^{(A)(0)}(y)\right]_{x_{0}=y_{0}}^{t r}$ contains gradient terms symmetric in the isospin indices [7]. The $[V, A]$-commutator does not lead to further restrictions. But there again appear gradient terms which are symmetric in the internal indices [8].

If we put according to (4):

$$
a=-b=i
$$

our axialvector current agrees up to the term linear in $A(x)$ with the mesonic part of the axialvector current of GELL-MANN and LEVY's $\sigma$-model [9].

\section{First Order Currents}

Within the $\boldsymbol{A}^{2} B^{2}$-theory we obtain from the Yang-Feldman equations for the first order field operators (for reasons of simplicity we take 
equal masses $m$ for the $A$-resp. $B$-particles):

$$
\begin{aligned}
& A_{i}^{(1)}(x)=\int d^{4} x^{\prime} \Delta_{R}\left(x-x^{\prime}\right): A_{i}^{(0)}\left(x^{\prime}\right) B^{(0)^{2}}\left(x^{\prime}\right): \\
& B^{(1)}(x)=\int d^{4} x^{\prime} \Delta_{R}\left(x-x^{\prime}\right): \boldsymbol{A}^{(0)^{2}}\left(x^{\prime}\right) B^{(0)}\left(x^{\prime}\right):
\end{aligned}
$$

With $Z_{A}^{(1)}=0$ we obtain by means of equation $(1),(2),(5)$ and the choice $a=-b=i$ for the first order currents:

$$
\begin{aligned}
j_{\mu i}^{(v)(1)}(x)= & \varepsilon_{i n m}: A_{n}^{(0)}(x) \overleftrightarrow{\partial_{\mu}^{x}} \int d^{4} x^{\prime} \Delta_{R}\left(x-x^{\prime}\right): A_{m}^{(0)}\left(x^{\prime}\right) B^{(0)^{2}}\left(x^{\prime}\right): \\
j_{\mu i}^{(A)(1)}(x)= & i \int d^{4} x^{\prime} \Delta_{R}\left(x-x^{\prime}\right) \overleftrightarrow{\partial_{\mu}^{x}}\left(: \boldsymbol{A}^{(0)^{2}}\left(x^{\prime}\right) A_{i}^{(0)}(x) B^{(0)}\left(x^{\prime}\right):\right. \\
& \left.-: A_{i}^{(0)}\left(x^{\prime}\right) B^{(0)^{2}}\left(x^{\prime}\right) B^{(0)}(x):\right)+c \partial_{\mu} A_{i}^{(1)}(x) .
\end{aligned}
$$

We note that due to their definition our currents contain no bilinear terms in the free fields in first order.

\section{First Order Commutators}

The first order commutators:

$$
\left[j_{\mu i}^{\alpha}(x), j_{\nu k}^{\beta}(y)\right]^{(1)}=\left[j_{\mu i}^{\alpha(0)}(x), j_{\nu k}^{\beta(1)}(y)\right]+\left[j_{\mu i}^{\alpha(1)}(x), j_{\nu k}^{\beta(0)}(y)\right]
$$

contain terms quadrilinear, trilinear, and bilinear in the free fields arising from single resp. double contractions.

The single contraction terms do not lead to any anormal result. Either two fields at the points $x$ and $y$ are contracted with each other leading to a $\Delta(x-y)$ function, whose time derivative gives at equal times normal, finite terms (those terms also contain gradient terms, as they are of the same canonical structure as the zeroth order commutators), or $x x^{\prime}$ resp. $y x^{\prime}$ contractions take place whose sum leads to the local chain:

$$
\begin{aligned}
\int d^{4} x^{\prime} P_{\mu \nu}\left(\partial^{x}, \partial^{y}\right) & \left(\Delta_{A}\left(x-x^{\prime}\right) \Delta_{A}\left(x^{\prime}-y\right)\right. \\
& \left.-\Delta_{R}\left(x-x^{\prime}\right) \Delta_{R}\left(x^{\prime}-y\right)\right): B^{(0)^{2}}\left(x^{\prime}\right):
\end{aligned}
$$

where $P_{\mu \nu}\left(\partial^{x}, \partial^{y}\right)$ is a second order polynomial in the derivatives. By means of contour integration in momentum space it is very easy to see that (7) vanishes for $x_{0}=y_{0}$.

Therefore we concentrate our effort on the bilinear terms which follow from double contractions. By means of straight-forward computation we obtain:

$$
\begin{gathered}
{\left[j_{\mu i}^{(V)}(x), j_{v k}^{(V)}(y)\right]_{\text {bilinear }}^{(1)}=\delta_{i k} \int d^{4} x^{\prime} I_{\mu \nu}\left(x, y, x^{\prime}\right): B^{(0)^{2}}\left(x^{\prime}\right):} \\
{\left[j_{\mu i}^{(A)}(x), j_{v k}^{(A)}(y)\right]_{\text {bilinear }}^{(1)}=-\frac{1}{2} \delta_{i k} \int d^{4} x^{\prime} I_{\mu \nu}\left(x, y, x^{\prime}\right)} \\
\left(: \boldsymbol{A}^{(0)^{2}}\left(x^{\prime}\right):+: B^{(0)^{2}}\left(x^{\prime}\right):\right) \\
{\left[j_{\mu i}^{(A)}(x), j_{\nu k}^{(V)}(y)\right]_{\text {bilinear }}^{(1)}=i \varepsilon_{i k r} \int d^{4} x^{\prime} A_{r}^{(0)}\left(x^{\prime}\right) B^{(0)}\left(x^{\prime}\right)} \\
I_{\mu \nu}\left(x, y, x^{\prime}\right)
\end{gathered}
$$


where $I_{\mu \nu}\left(x, y, x^{\prime}\right)$ is defined as follows:

$$
\begin{aligned}
& I_{\mu v}\left(x, y, x^{\prime}\right) \equiv-i\left\{-\left[\Delta^{(1)}\left(y-x^{\prime}\right) \Delta_{A}\left(x^{\prime}-x\right)+\Delta_{R}\left(y-x^{\prime}\right) \Delta^{(1)}\left(x^{\prime}-x\right)\right]\right. \\
& \cdot \overleftrightarrow{\partial_{\mu}^{x}} \stackrel{\leftrightarrow}{\partial_{\nu}^{y}} \Delta(x-y)+\left[\Delta_{A}\left(y-x^{\prime}\right) \Delta_{A}\left(x^{\prime}-x\right)-\Delta_{R}\left(y-x^{\prime}\right) \Delta_{R}\left(x^{\prime}-x\right)\right] \\
& \left.. \overleftrightarrow{\partial_{\mu}^{x}} \stackrel{\leftrightarrow}{\partial_{\nu}^{v}} \Delta^{(1)}(x-y)\right\} .
\end{aligned}
$$

As the vector current is conserved, $I_{\mu \nu}$ is divergenceless, i. e.:

$$
\partial_{x}^{\mu} I_{\mu \nu}\left(x, y, x^{\prime}\right)=\partial_{y}^{v} I_{\mu \nu}\left(x, y, x^{\prime}\right)=0
$$

It is amusing to note that according to equation (9) and (10) our axialvector current is effectively conserved in the bilinear part of the commutators.

\section{Jost-Lehmann-Dyson Representation for First Order Commutators}

Let us consider $I_{\mu \nu}$ in momentum space. We define:

$$
\tilde{I}_{\mu \nu}(q, \Delta)=\int d^{4} x^{\prime} e^{-i \Delta x^{\prime}} \int d^{4} \zeta e^{i q \zeta} I_{\mu \nu}\left(\frac{\zeta}{2},-\frac{\zeta}{2}, x^{\prime}\right) .
$$

Inserting (10) into (11) one obtains immediately:

$$
\begin{aligned}
& \tilde{I}_{\mu \nu}(q, \Delta)=(2 \pi)^{-2} \int d^{4} k \delta\left(k^{2}-\mu^{2}\right)\left(2 k-q+\frac{\Delta}{2}\right)_{\nu}\left(2 k-q-\frac{\Delta}{2}\right)_{\mu} \\
& \cdot\left\{\frac{P}{\mu^{2}-\left(k-q-\frac{\Delta}{2}\right)^{2}} \delta\left(\left(k-q+\frac{\Delta}{2}\right)^{2}-\mu^{2}\right)\left[\varepsilon(k)-\varepsilon\left(k-q+\frac{\Delta}{2}\right)\right]\right. \\
& +f(\Delta \rightarrow-\Delta)+i \pi \varepsilon(k) \delta\left(\left(k-q+\frac{\Delta}{2}\right)^{2}-\mu^{2}\right) \delta\left(\left(k-q-\frac{\Delta}{2}\right)^{2}-\mu^{2}\right) \\
& \left.\cdot\left[\varepsilon\left(k-q+\frac{\Delta}{2}\right)-\varepsilon\left(k-q-\frac{\Delta}{2}\right)\right]\right\} .
\end{aligned}
$$

The imaginary part (i. e. the last term) of eq. (12) vanishes for $\Delta^{2}<4 \mu^{2}$. In the following we concentrate our effort on this case exclusively.

Then we may rewrite (12) into the form

$$
\begin{aligned}
& \tilde{I}_{\mu \nu}(q, \Delta)=-i(2 \pi)^{-3} \int d^{4} k \delta\left(k^{2}-\mu^{2}\right)
\end{aligned}
$$

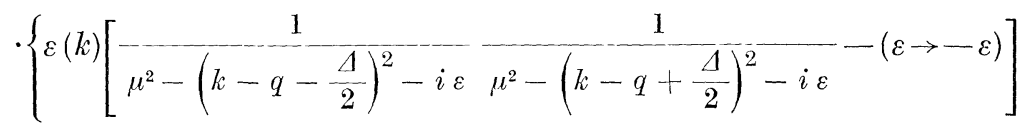

$$
\begin{aligned}
& \left.-\left[\frac{1}{\mu^{2}-\left(k-q-\frac{\Delta}{2}+i \varepsilon\right)^{2}} \frac{1}{\mu^{2}-\left(k-q+\frac{\Delta}{2}+i \varepsilon\right)^{2}}-(\varepsilon \rightarrow-\varepsilon)\right]\right\}
\end{aligned}
$$


Now we apply the formula ${ }^{2}$

with

$$
\frac{1}{\mu^{2}-\left(p-\frac{\Delta}{2}\right)^{2}} \frac{1}{\mu^{2}-\left(p+\frac{\Delta}{2}\right)^{2}}=\int d^{4} u \int d s \frac{\varrho(s, u, \Delta)}{s-(p-u)}
$$

where

$$
\begin{aligned}
\varrho(s, u, \Delta)= & \frac{\partial}{\partial s}\left\{\left[\delta\left(u+\frac{\Delta}{2}-\phi\left(s, \Delta^{2}\right) \Delta\right)+(\Delta \rightarrow-\Delta)\right]\right. \\
& \cdot \theta\left(\phi\left(s, \Delta^{2}\right)\right) \theta\left(\left(1-\phi\left(s, \Delta^{2}\right)\right)\left|\phi^{\prime}\left(s, \Delta^{2}\right)\right|\right\}
\end{aligned}
$$

and obtain for (12)

$$
\phi\left(s, \Delta^{2}\right)=\frac{1}{2} \sqrt{\frac{1}{4}+\frac{s-\mu^{2}}{\Delta^{2}}}
$$

$$
\begin{aligned}
& \tilde{I}_{\mu \nu}(q, \Delta)=(2 \pi)^{-2} \int d^{4} k \delta\left(k^{2}-\mu^{2}\right) \int d^{4} u \int d s \\
& {[\varepsilon(k)-\varepsilon(k-q-u)] \delta\left(s-(k-q-u)^{2}\right) \varrho(s, u, \Delta)} \\
& \left(2 k-q-\frac{\Delta}{2}\right)_{\mu}\left(2 k-q+\frac{\Delta}{2}\right)_{\nu} .
\end{aligned}
$$

The $k$-integration in (17) may be done by means of standard techniques [10].

In this way we get finally the following Dyson-representation ${ }^{3}$ for $I_{u v}(q, \Delta)$ :

$$
\begin{aligned}
\tilde{I}_{\mu \nu}(q, \Delta)= & (2 \pi)^{-2} \int d^{4} u \int d s \varepsilon(q+u) \delta\left(s-(q+u)^{2}\right) \\
& \cdot\left\{g_{\mu^{\nu}} \psi_{1}(s, u, \Delta)+(q+u)_{\mu}(q+u)_{\nu} \psi_{2}(s, u, \Delta)\right. \\
& +\left(u_{\mu} u_{\nu}-\frac{1}{4} \Delta_{\mu} \Delta_{\nu}\right) \psi_{3}(s, u, \Delta) \\
& \left.+\left[(q+u)_{\mu}\left(u+\frac{\Delta}{2}\right)_{\nu}+(q+u)_{\nu}\left(u-\frac{\Delta}{2}\right)_{\mu}\right] \psi_{4}(s, u, \Delta)\right\}
\end{aligned}
$$

with

$$
\begin{aligned}
& \psi_{1}(s, u, \Delta)=\int d s^{\prime} \varrho\left(s^{\prime}, u, \Delta\right) f\left(s, s^{\prime}\right) \frac{1}{3 s}\left[-\left(\mu^{2}-s^{\prime}\right)^{2}+s\left(2\left(\mu^{2}+s^{\prime}\right)-s\right)\right] \\
& \psi_{2}=\int d s^{\prime} \varrho\left(s^{\prime}, u, \Delta\right) f\left(s, s^{\prime}\right) \frac{1}{3 s^{2}}\left[s^{2}-2 s\left(\mu^{2}+s^{\prime}\right)+4\left(\mu^{2}-s^{\prime}\right)^{2}\right] \\
& \psi_{3}=\int d s^{\prime} \varrho\left(s^{\prime}, u, \Delta\right) f\left(s, s^{\prime}\right) . \\
& \psi_{4}=\int d s^{\prime} \varrho\left(s^{\prime}, u, \Delta\right) f\left(s, s^{\prime}\right) \frac{\mu^{2}-s^{\prime}}{s}
\end{aligned}
$$

${ }^{2}$ Eq. (14) may be derived easily by means of Feynman's identity $\frac{1}{a \cdot b}$ $=\int_{0}^{1} d \alpha \frac{1}{[a \alpha+b(1-\alpha)]^{2}}$.

${ }^{3}$ After performing the $u$-integration by means of the $\delta$-function in (15) and having introduced the substitutions $s^{\prime} \rightarrow x \equiv \phi-1 / 2$, equation (18) becomes a Dyson-representation of the vertical type [11].

23 Commun. math. Phys., Vol. 8 
and

$$
f(x, s)=\frac{\pi}{x}\left[\left(x-(\sqrt{s}+\mu)^{2}\right)\left(x-(\sqrt{s}-\mu)^{2}\right)\right]^{1 / 2} \theta\left(x-(\sqrt{ } s+\mu)^{2}\right) .
$$

We note two important properties of the spectral functions $\psi_{i}(u, s)$ :

a) Asymptotic behaviour in $s$ :

Due to the fact, that $\varrho\left(s^{\prime}\right)=\frac{\partial}{\partial s^{\prime}} \hat{\varrho}\left(s^{\prime}\right)$ and $\operatorname{supp} \hat{\varrho}\left(s^{\prime}\right)$ is compact follows $\psi_{i}(u, s) \underset{s \rightarrow \infty}{\sim} 0\left(s^{-1}\right) \quad i=2,3,4$ and $\psi_{1}(u, s)_{s \rightarrow \infty}^{\sim} 0(1)$

b) Symmetry in $u$ :

$$
\psi_{i}(s, u)=\psi_{i}(s,-u)
$$

\section{Equal Time Commutators}

In the following we collect the main arguments in the final evaluation of the ETCR starting from the Dyson representation Eq. (18).

$$
\text { A. } \mu=\nu=0
$$

Contribution from $\psi_{1}$ and $\psi_{2}$ : We have $\psi_{1}(s, u, \Delta) \underset{s \rightarrow \infty}{\longrightarrow}-\pi \int d s^{\prime}$ $\cdot \hat{\varrho}\left(s^{\prime}, u, \Delta\right)+0\left(s^{-1}\right) \quad \psi_{2} \underset{s \rightarrow \infty}{\longrightarrow}-\frac{1}{s} \psi_{1}$.

Therefore:

$$
\left.\psi_{1}(q+u)^{2}, u, \Delta\right)+\left(q_{0}+u_{0}\right) \psi_{2}\left((q+u)^{2}, u, \Delta\right) \underset{q_{0} \rightarrow \infty}{\longrightarrow} 0\left(\left(q_{0}+u_{0}\right)^{-2}\right)
$$

i. e. due to $\varepsilon\left(q_{0}+u_{0}\right)$ we have no contribution from $\psi_{1,2}$ to

$$
\lim _{T \rightarrow 0} \int_{-1 / T}^{1 / T} \tilde{I}_{00}(q, \Delta) d q_{0} .
$$

Contribution from $\psi_{3}$ : As $\psi_{3}(s, u, \Delta) \underset{s \rightarrow \infty}{\sim} 0\left(s^{-1}\right)$ the same argument as above may be applied.

Contribution from $\psi_{4}$ : This contribution may be written as:

with :

$$
\begin{aligned}
& \Delta_{0} \lim _{T \rightarrow 0} \int_{-1 / T}^{1 / T} d q_{0} \int_{-1 / 2}^{+1 / 2} d x \varkappa\left(q_{0}+\Delta_{0} x\right) \varepsilon\left(q_{0}+\Delta_{0} x\right) \\
& \cdot \theta\left((q+\Delta x)^{2}-\left(\sqrt{s^{\prime}(x)}+\mu\right)^{2}\right) g\left((q+\Delta x)^{2}, s^{\prime}(x)\right)
\end{aligned}
$$

$g$ is defined as:

$$
s^{\prime}(x)=\Delta^{2}\left(x^{2}-\frac{1}{4}\right)+\mu^{2}
$$

$$
g\left(x, s^{\prime}(x)\right)=\frac{\partial}{\partial s^{\prime}}\left(f\left(x, s^{\prime}(x)\right) \frac{\mu^{2}-s^{\prime}(x)}{x}\right)
$$

and has the asymptotic behaviour

$$
g\left(x, s^{\prime}(x)\right) \underset{x \rightarrow \infty}{\longrightarrow} 0\left(x^{-1}\right) .
$$


Now we have by a change of variable:

$$
\begin{aligned}
\int_{-1 / T^{\prime}}^{1 / T} d q_{0}\left(q_{0}+\Delta_{0} \varkappa\right) \varepsilon\left(q_{0}+\Delta_{0} \varkappa\right) \theta\left((q+\Delta \varkappa)^{2}-\left(\sqrt{s^{\prime}(\varkappa)}+\mu\right)^{2}\right) \\
\cdot g\left((q+\Delta \varkappa)^{2}, s^{\prime}(\varkappa)\right) \\
=\frac{1}{2}\left(\int_{0}^{B_{+}(\varkappa)}+\int_{0}^{B_{-}(x)}\right) d \zeta g\left(\zeta+\left(\sqrt{s^{\prime}(\varkappa)}+\mu\right)^{2}, s^{\prime}(\varkappa)\right)
\end{aligned}
$$

with

$$
B_{ \pm}(x)=\left(\frac{1}{T} \pm \Delta_{0} x\right)^{2}-\left(\boldsymbol{q}+\boldsymbol{\Delta}^{x}\right)^{2}-\left(\sqrt{s^{\prime}(x)}+\mu\right)^{2} .
$$

But only that part of (22) contributes to (21) which is antisymmetric in $x$, i. e. we may use instead of $(22)$ the antisymmetrized expression:

$$
\frac{1}{4}\left(\int_{B_{+}(-x)}^{B_{+}(x)}+\int_{B_{-}(-\varkappa)}^{B_{-}(\varkappa)}\right) d \zeta g\left(\zeta+\left(\sqrt{s^{\prime}(\varkappa)}+\mu\right)^{2}, s^{\prime}(\varkappa)\right) \underset{T \rightarrow 0}{\longrightarrow} 0 .
$$

Therefore we conclude:

$$
\lim _{T \rightarrow 0} \int_{-1 / T}^{1 / T} d q_{0} \tilde{I}_{00}(q, \Delta)=0
$$

i. e. the ETCR for $\mu=v=0$ gives a vanishing contribution in agreement with Gell-Mann's conjecture.

$$
\text { B. } \mu=0, v=\rightarrow
$$

1. $\psi_{1}$ gives no contribution.

2. $\left(q_{0}+u_{0}\right) \boldsymbol{u} \psi_{2,4}$ gives no contribution due to $\boldsymbol{u}=\boldsymbol{\Delta} \varkappa$ and applying the same arguments as above.

3 . Contributions arising from the terms

$$
\left(u_{0} \boldsymbol{u}-\frac{1}{4} \Lambda_{0} \boldsymbol{\Delta}\right) \psi_{3},(\boldsymbol{q}+\boldsymbol{u})\left(u_{0}-\frac{\Delta_{0}}{2}\right) \psi_{4}:
$$

Due to the asymptotic behaviour of $\psi_{3,4}$ and the symmetric integration over $q_{0}$ we obtain no contribution.

4. There remain the terms

$$
\begin{aligned}
& \left(q_{0}+u_{0}\right)\left(\boldsymbol{q} \psi_{2}+\frac{\boldsymbol{\Delta}}{2} \psi_{4}\right) \\
\equiv & \left(q_{0}+u_{0}\right)\left(\boldsymbol{q}+\frac{\boldsymbol{\Delta}}{2}\right) \psi_{4}+\left(q_{0}+u_{0}\right) \boldsymbol{q}\left(\psi_{2}-\psi_{4}\right) .
\end{aligned}
$$

As $\psi_{4} \underset{s \rightarrow \infty}{\longrightarrow} 0\left(s^{-1}\right)$ (due to the form of $\psi_{4}$ this asymptotic behaviour does not become better after performing the $u$-integration!) the first term in (23) leads for the ETCR to a logarithmic divergent gradient term, $23^{*}$ 
i. e. in configuration space it looks like:

$$
\sim \lim _{T \rightarrow 0} \ln (T) \operatorname{grad}_{x} \delta(\boldsymbol{x}-\boldsymbol{y}) .
$$

The second term in (23) gives a vanishing ETCR-contribution due to the following arguments:

1. $\psi_{2}-\psi_{4} \underset{s \rightarrow \infty}{\widetilde{\sim}} 0\left(s^{-2}\right)$ because with the asymptotic expansion:

$$
f\left(s, s^{\prime}\right) \underset{s \rightarrow \infty}{\sim} \pi\left(1-\frac{\mu^{2}+s^{\prime}}{s}+0\left(s^{-1}\right)\right)
$$

one sees immediately, that

$$
\frac{\partial}{\partial s^{\prime}}\left[\frac{f\left(s, s^{\prime}\right)}{s^{2}}\left(s^{2}+4\left(\mu^{2}-s^{\prime}\right)^{2}-5 s \mu^{2}+s s^{\prime}\right)\right]_{s \rightarrow \infty} 0\left(s^{-2}\right) .
$$

2. Due to this asymptotic behaviour, the ETCR-contribution of the 2 nd term in (23) may be written as:

$$
\boldsymbol{q} \int d^{4} u \int d s \psi_{2}(s, u, \Delta)-\psi_{4}(s, u, \Delta) .
$$

Now, an explicit evaluation shows that:

$$
\int d s \frac{\partial}{\partial s^{\prime}}\left[\frac{f\left(s, s^{\prime}\right)}{s^{2}}\left(s^{2}+4\left(\mu^{2}-s^{\prime}\right)^{2}-5 s \mu^{2}+s s^{\prime}\right)\right]=0
$$

i. e. (24) vanishes.

$$
\text { C. } \mu, v \text { space-like }
$$

Due to the symmetric $q_{0}$-integration and the asymptotic behaviour of the $\psi_{i}$ we can only have a non-vanishing ETCR-contribution for $\mu=v$ arising from $\psi_{1}$. But also this contribution vanishes due to the symmetry of $\psi_{1}$ for $u \rightarrow-u$.

In all these computations of equal time commutators we have performed a formal symmetric $q_{0}$ integration. The use of a symmetric interpolating test function of the type proposed in the introduction does not change the results obtained by the more formal integration method.

\section{Conclusions}

In the case of the coupling $\boldsymbol{A}^{2}(x) \boldsymbol{B}^{2}(x)$ we found logarithmic divergent gradient terms for the ETCR of the zero-space components of currentcurrent commutators. It is easily seen that the same results hold for the other quadrilinear couplings.

Concerning the trilinear coupling $\boldsymbol{A}^{2}(x) B(x)$ the linear terms in the current-current commutators may be expressed by $I_{\mu \nu}$ too. But then we have to consider in momentum space $\tilde{I}_{\mu \nu}(q, \Delta)$ for time-like $\Delta\left(\Delta^{2}\right.$ $\left.=\mu^{2}\right)$. Again we obtain the same results as in case of quadrilinear couplings, because our Dyson-representation Eq. (18) for the commutator matrix element is correct for all $\Delta^{2}$ with $\Delta^{2}<4 \mu^{2}$. 
In case of the quadrilinear couplings one would also be interested to learn something on the bilinear contributions to the ETCR for timelike $\Delta$ (i. e. $\Delta^{2}>4 \mu^{2}$ ). But this would require an explicit analytic continuation of our Dyson-representation in $\Delta^{2}$, which is a somewhat involved task. We, therefore, dispense with this.

The isospin-dependence of the divergent terms we have found agrees with the general conjecture of AdLer and CALLaN [7]. To be more specific, we find ( $\alpha_{i}$ denotes the kind of current)

$$
\left\{\left[j_{\mu i}^{\alpha_{1}}(x), j_{v k}^{\alpha_{2}}(y)\right]^{(1)}-\left[j_{\mu k}^{\alpha_{2}}(x), j_{v i}^{\alpha_{1}}(y)\right]^{(1)}\right\}_{\text {bilinear }}=0
$$

already for finite time separations.

It would be interesting to check, whether in case of our models the Adler-Callan conjecture is true in higher orders of perturbation theory. This question will be discussed by us in the near future.

Note added in proof. After completion of our paper we received a preprint by N. Usyukixa from Dubna, where similar methods have been applied to ETCR within the Johnson-Low model.

\section{References}

1. Schroer, B., and P. Sticher: Commun. Math. Phys. 3, 258 (1966).

2. Johnson, K., and F. E. Low: Progr. Theor. Phys. Suppl. 37, 38, 74 (1966).

3. Hamprecht, B.: Nuovo Cimento 47 A, 770 (1967) and 50 A, 449 (1967). Polkinghorne, J. C.: Nuovo Cimento 52 A, 351 (1967). We do not quote papers, where primarily sum rules and not ETCR are checked in perturbation theory.

4. Langerholc, J.: DESY 67/26, August 1967.

5. Kuo, T. K., and M. Sugawara: Phys. Rev. 151, 1181 (1966).

6. Adler, S. L., and C. G. Callax: CERN, TH. 587 (1965). - Kramer, G., and K. MeEtz: DESY 67/11, April 1967.

7. Gell-Manx, M.: Phys. Rev. 125, 1067 (1962).

8. Adler, S. L., and C. G. Callan: CERN, TH. 587 (1965).

9. Gell-Mann, M., and M. Levy: Nuovo Cimento 16, 705 (1960).

10. Compare chapter VI in G. KäLLEN's review article on Quantum Electrodynamics in: Encyclopedia of Physics, Vol. V, Part 1. Berlin-GöttingenHeidelberg: Springer 1958.

11. Compare: Kramer, G., and K. Meetz: Commun. Math. Phys. 3, 29 (1966).

Professor Dr. P. Stichel

Physikalisches Staatsinstitut

IJ. Inst. f. Experimentalphysik

2000 Hamburg 50

Luruper Chaussee 149 